\title{
Efficacy of various dietary calcium salts to improve intestinal resistance to Salmonella infection in rats
}

\author{
Sandra J. M. ten Bruggencate ${ }^{1}$, Johannes Snel ${ }^{1 *}$, Margriet H. C. Schoterman ${ }^{2}$, Ellen Ertmann ${ }^{2}$, \\ Esther van der Meulen ${ }^{1}$, Arjan Schonewille ${ }^{1}$ and Ingeborg M. J. Bovee-Oudenhoven ${ }^{1,3}$ \\ ${ }^{1}$ NIZO Food Research, Ede, The Netherlands \\ ${ }^{2}$ FrieslandCampina Domo, Zwolle, The Netherlands \\ ${ }^{3}$ TI Food and Nutrition, Nutrition and Health Program, Wageningen, The Netherlands
}

(Received 16 March 2010 - Revised 26 July 2010 - Accepted 25 August 2010 - First published online 29 September 2010)

\section{Abstract}

Previous animal and human studies have shown protective effects of Ca on the resistance to enteropathogenic infections. Most interventions were performed with calcium phosphate and little is known about the protective effect of other dietary sources of Ca. Therefore, we investigated the efficacy of several Ca salts to enhance intestinal resistance to Salmonella enteritidis infection. Rats ( $n 7-8$ per group) were fed a high-fat, Western human-style, purified diet with a low Ca content ( $20 \mathrm{mmol}$ calcium phosphate/kg; negative control group) or the same diet supplemented with either (extra) calcium phosphate, milk Ca, calcium chloride or calcium carbonate (total of $100 \mathrm{mmol}$ Ca supplement $/ \mathrm{kg}$ ). Diets contained Cr-EDTA for assessment of incremental changes in intestinal permeability. After an adaptation period of 2 weeks, animals were orally infected with $S$. enteritidis to mimic a human-relevant foodborne infection. Ca supplement-induced changes on faecal lactobacilli and enterobacteria were studied before infection. Changes in intestinal permeability were determined by measuring urinary $\mathrm{Cr}$ with time. Persistence of Salmonella was determined by studying faecal excretion of this pathogen in time. Overall, all Ca salts increased resistance towards Salmonella. After infection, body weight gain and food intake were higher in the calcium phosphate group. Calcium phosphate and milk Ca decreased faecal enterobacteria before infection. All Ca salts decreased infection-induced intestinal permeability and persistence of Salmonella. Calcium phosphate, milk Ca, calcium carbonate and calcium chloride are able to enhance the intestinal resistance to Salmonella in rats.

Key words: Salmonella: Rats: Infection: Intestinal permeability: Calcium salts: Dairy products: Enterobacteria

Gastrointestinal infections are still a major health problem, not only in developing countries ${ }^{(1,2)}$. The enteric pathogen Salmonella enteritidis is one of the leading causes of gastrointestinal infections in humans, ranging from mild, self-limiting diarrhoea and/or inflammation of the intestinal mucosa to life-threatening systemic infection ${ }^{(3,4)}$. The growing resistance of pathogenic bacteria to antibiotic drugs $^{(4)}$ makes it important to develop ways to prevent and treat intestinal infections by other means ${ }^{(5)}$. Besides hygienic measures, dietary modulation of host resistance to intestinal infections might be an attractive approach. By influencing the composition of gastrointestinal contents, diet affects the gastrointestinal survival of pathogens ${ }^{(6,7)}$, the composition of autochthonous intestinal microbiota ${ }^{(8,9)}$ and the epithelial barrier function ${ }^{(10)}$. These primary nonimmunological host defence mechanisms of the gastrointestinal tract are particularly important in withstanding the first encounter with a pathogen.
Dietary Ca has been recognised to increase resistance by decreasing colonisation and translocation of common intestinal Gram-negative pathogens, both in rats ${ }^{(8,11)}$ and in humans ${ }^{(12)}$. We hypothesise that this resistance-enhancing effect of calcium phosphate might be explained by three potential mechanisms. First, it is known that intake of $\mathrm{Ca}$ and phosphate results in the formation of an amorphous calcium phosphate complex that adsorbs and precipitates luminal cytotoxic components, such as bile acids and fatty acids. This can subsequently stimulate growth of protective members of the endogenous microbiota ${ }^{(8)}$, which exerts antagonistic activity towards foodborne pathogens. Second, Salmonella is a pathogenic microorganism that can translocate to extra-intestinal organs such as the liver and spleen. Amorphous calcium phosphate-induced precipitation of cytotoxic components reduces epithelial cell damage ${ }^{(13)}$ which might strengthen gut barrier function and reduce translocation of 
Salmonella. Finally, amorphous calcium phosphate might bind Salmonella in the intestinal lumen, thereby preventing Salmonella adherence to the gut mucosa.

Until now, dietary intervention studies have focused on calcium phosphate. From the above-mentioned studies, it can be argued that the combination and luminal interaction of dietary $\mathrm{Ca}$ with phosphate is necessary for the resistance-enhancing effect as the adsorbing (binding) capacity of amorphous calcium phosphate is much larger than that of other $\mathrm{Ca}$ salts ${ }^{(14)}$. Little is known about the in vivo effects of other Ca salts on resistance to Salmonella. Considering the high phosphate content of human ${ }^{(15)}$ and rodent $^{(16)}$ diets, the amorphous calcium phosphate could also be formed with other dietary Ca salts. Therefore, we tested the efficacy of several dietary Ca salts to increase the resistance of rats to Salmonella infection. In addition, in vitro experiments on $\mathrm{pH}$-dependent solubility of the various $\mathrm{Ca}$ salts and their capacity to bind Salmonella were determined to gain further mechanistic insight.

\section{Materials and methods}

\section{In vitro experiments}

Solubility of calcium salts. Only ionic Ca can bind to phosphate and form the amorphous calcium phosphate complex in the gut lumen. Therefore, we studied the solubility of the various Ca salts in the gut lumen. Solubility of the following Ca salts was investigated: calcium phosphate (CaHPO ; purity $>98 \%$; Merck, Darmstadt, Germany), whey product rich in milk Ca $(29 \% \mathrm{Ca}$, other minerals and milk salts $66 \%$; Vivinal ${ }^{\circledR}$ MCA FrieslandCampina Domo, Zwolle, The Netherlands) further referred to as milk Ca, calcium carbonate (purity $>99 \%$; Calcitec V/ 40S, Mineraria, Italy) or calcium chloride (purity $>99 \%$; Merck). These were tested at a final $\mathrm{Ca}$ concentration of $40 \mathrm{~mm}$ and at different $\mathrm{pH}$ levels representing the $\mathrm{pH}$ range of the gastrointestinal tract.The different $\mathrm{pH}$ levels ranging from 2 to 7 were achieved by either adding a glycine buffer (250 mm, pH 2 and 3), an acetate buffer (250 mm, pH 4 and 5) or a 3-( $N$-morpholino) propanesulphonic acid buffer $(250 \mathrm{~mm}, \mathrm{pH} 6$ and 7$)$. After $1 \mathrm{~h}$ incubation at $37^{\circ} \mathrm{C}$, samples were centrifuged at $18000 \mathrm{~g}$ for $5 \mathrm{~min}$ and supernatants were diluted in $0.5 \mathrm{~g} / \mathrm{l} \mathrm{CsCl}$. $\mathrm{Ca}$ concentrations were determined in the diluted supernatants by inductive coupled plasma-atomic emission spectrophotometry (Varian, Mulgrave, VIC, Australia).

Binding of Salmonella. In order to test whether the amorphous calcium phosphate complex is able to precipitate Salmonella in the gut lumen (in contrast to crystalline calcium phosphate or ionic $\mathrm{Ca}$ ), we adapted a method that has been previously described to study precipitation of bile salts ${ }^{(14)}$. Increasing concentrations (final $0-40 \mathrm{~mm}$ ) of amorphous calcium phosphate, crystalline calcium phosphate or ionic Ca in HEPES buffer $(500 \mathrm{~mm})$ were added to a Salmonella suspension in saline $\left(10^{9}\right.$ colony-forming units/ml). Amorphous calcium phosphate was freshly formed as described elsewhere ${ }^{(14)}$. In short, equimolar amounts of dissolved calcium chloride and sodium hydrogen phosphate were mixed to form the amorphous calcium phosphate complex. After $15 \mathrm{~min}$ incubation at $37^{\circ} \mathrm{C}$, samples were centrifuged for $2 \mathrm{~min}$ at $500 \mathrm{~g}$. The supernatants were sonicated for $1 \mathrm{~min}$ using the Sonicator Ultrasonic Processor XL (Heat Systems, Farmingdale, NY, USA) to disintegrate bacterial cell walls. Total protein, a sensitive way to quantify bacterial content, was determined using the Bradford protein assay (Interchim, Montlucon, France).

\section{In vivo experiment}

Animals and diet. The experimental protocol was approved by the animal welfare committee of Wageningen University (Wageningen, The Netherlands). Specific pathogen-free, male, young adult (8 weeks old) Wistar rats with a mean body weight of $276 \mathrm{~g}$ (WU, Harlan, Horst, The Netherlands) were housed individually in metabolism cages. All the rats were kept in a temperature$\left(22-24^{\circ} \mathrm{C}\right)$ and humidity-controlled environment (50-60\%) with a $12 \mathrm{~h}$ light-dark cycle. To study the effects of the various dietary Ca salts on the main effect variables intestinal permeability and Salmonella persistence, the rats were randomly assigned to either an experimental diet with a total of $20 \mathrm{mmol} / \mathrm{kg}$ calcium phosphate (low-Ca negativecontrol group) or diets supplemented to $100 \mathrm{mmol} \mathrm{Ca} / \mathrm{kg}$ with calcium phosphate $\left(\mathrm{CaHPO}_{4}\right.$; purity $>98 \%$; Merck), milk Ca (a whey-derived product; $29 \% \mathrm{Ca}$, other minerals and milk salts $66 \%$; Vivinal ${ }^{\circledR}$ MCA FrieslandCampina Domo), calcium carbonate (purity $>99 \%$; Calcitec V/40S, Mineraria) or calcium chloride (purity $>99 \%$; Merck). Diets and demineralised drinking water were supplied ad libitum. Compared with the AIN-93 recommendation for rat diets ${ }^{(16)}$, the diets had a high fat content $(200 \mathrm{~g}$ fat $/ \mathrm{kg}$ provided by $80 \mathrm{~g} / \mathrm{kg}$ maize oil and $120 \mathrm{~g} / \mathrm{kg}$ palm oil) to mimic the composition of a Western human diet. The composition of the diets has been described earlier ${ }^{(17)}$. Food intake and body weight were measured every $2-3 \mathrm{~d}$ before infection and daily after infection. After infection, the average of daily food intake $(\mathrm{kJ} / \mathrm{d})$ and growth was calculated per animal.

Calcium excretion. Ca was determined in freeze-dried faeces after dry-ashing and destruction. Faeces were treated with $50 \mathrm{~g} / 1$ of TCA $(1: 1, \mathrm{v} / \mathrm{v})$ and centrifuged for $2 \mathrm{~min}$ at $14000 \mathrm{~g}$. The supernatants were diluted with $0.5 \mathrm{~g} / 1 \mathrm{CsCl}$ and analysed by inductive coupled plasmaatomic emission spectrophotometry (Varian).

Urine was treated with $50 \mathrm{~g} / \mathrm{l}$ of TCA (1:1, v/v) and centrifuged for $2 \mathrm{~min}$ at $14000 \mathrm{~g}$. The supernatants were analysed as described for faeces. Since daily feed intake differed between animals and between dietary groups, faecal and urinary $\mathrm{Ca}$ excretion was calculated as percentage of intake. 
Composition of the intestinal microbiota. Fresh faecal samples collected $1 \mathrm{~d}$ before infection were analysed for the number of lactobacilli and enterobacteria. Faecal lactobacilli were quantified by plating appropriate 10 -fold dilutions on Rogosa agar (Oxoid, prepared according to instructions of the manufacturer) and incubating the plates in an anaerobic cabinet (Coy Laboratory Products, Inc., Ann Arbor, MI, USA) under an anaerobic gas mixture $\left(85 \% \mathrm{~N}_{2}, 10 \% \mathrm{CO}_{2}\right.$ and $\left.5 \% \mathrm{H}_{2}\right)$ at $37^{\circ} \mathrm{C}$ for $3 \mathrm{~d}$ as described previously ${ }^{(18)}$. Enterobacteria were determined by plating 10-fold dilutions on Levine EMB agar (Difco Laboratories, Detroit, MI, USA) and incubating overnight at $37^{\circ} \mathrm{C}^{(18)}$.

Intestinal permeability. In order to measure intestinal permeability, the marker Cr-EDTA was added to all the diets. Cr-EDTA, an inert complex that is not actively taken up by the mucosa ${ }^{(19,20)}$, was prepared as described elsewhere $^{(21)}$. Total $24 \mathrm{~h}$ urine samples were collected on the last day before and on 7 consecutive days after oral infection of the rats. Oxytetracycline $(1 \mathrm{mg})$ was added to the urine collection vessels of the metabolic cages daily to prevent bacterial deterioration. To measure Cr-EDTA, urine was treated with $50 \mathrm{~g} / 1$ of TCA $(1: 1, \mathrm{v} / \mathrm{v})$ and centrifuged for $2 \mathrm{~min}$ at $14000 \mathrm{~g}$. The supernatants were diluted with $0.5 \mathrm{~g} / \mathrm{l} \mathrm{CsCl}$ and $\mathrm{Cr}$ was analysed by inductive coupled plasma-atomic emission spectrophotometry. Urinary CrEDTA excretion was calculated as a percentage of daily dietary Cr-EDTA intake.

Oral infection with Salmonella. After adaptation to the housing and dietary conditions for 2 weeks, the rats were orally infected by gastric gavage of $10^{9}$ colony-forming units of S. enteritidis (clinical isolate, phage type 4 according to international standards; B1241 culture of NIZO food research, Ede, the Netherlands) suspended in $1 \mathrm{ml}$ of saline. Stability of the infection stock solution was determined by plating serial dilutions on Modified Brilliant Green Agar (Oxoid) containing sulphmandelate (Oxoid) immediately before and after the oral dosing. Salmonella was cultured and stored as described earlier ${ }^{(22)}$. On day 10 after oral infection, the rats were killed by $\mathrm{CO}_{2}$ inhalation.

Persistence of Salmonella. Immediately before and on days 1, 2, 5 and 7 after $S$. enteritidis infection, fresh faecal samples were collected directly from the anus of the animals and analysed for viable Salmonella by plating 10-fold dilutions on Modified Brilliant Green Agar (Oxoid) containing sulphmandelate (Oxoid) and incubating overnight at $37^{\circ} \mathrm{C}$.

Statistical analysis. Results are expressed as means with their standard errors, $n 7$ in the calcium phosphate group and $n 8$ in the other groups. One animal from the calcium phosphate group was excluded from all the results because of oral-pharyngeal reflux of the Salmonella suspension resulting in pneumonia. A commercially available package (Statistica 6.1; StatSoft, Inc., Tulsa, OK, USA) was used for all statistics.

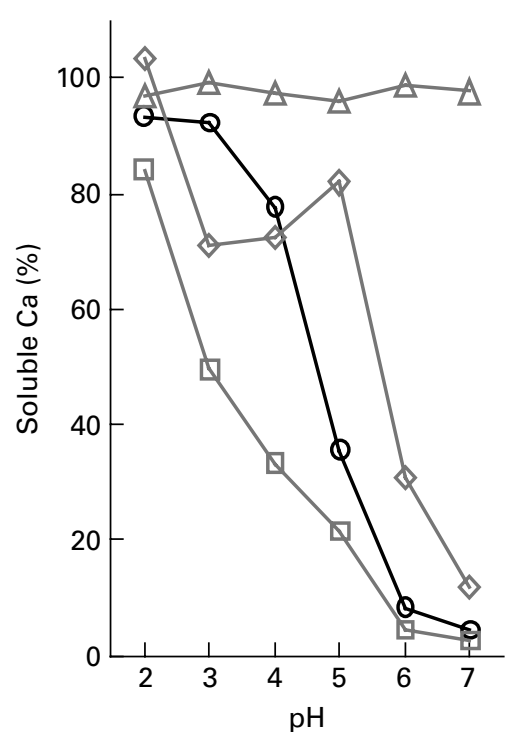

Fig. 1. Solubility of different $\mathrm{Ca}$ salts as a function of $\mathrm{pH}$. Values are exemplary for a few studies with different Ca salts. $\bigcirc$, Calcium phosphate; $\square$, milk $\mathrm{Ca} ; \diamond$, Ca carbonate; $\triangle$, calcium chloride.

All dietary Ca groups were only compared to the low-Ca control group. In case of normally distributed data (as indicated by the Shapiro-Wilk test), differences between means were tested for their significance using a one-way ANOVA, followed by the Student $t$ test (two sided). When data were not normally distributed, differences were tested for their significance using a Kruskall-Wallis ANOVA, followed by the non-parametric Mann-Whitney $U$ test (two sided). Repeated-measures ANOVA was used for intestinal permeability and Salmonella colonisation. Bonferroni correction was used for multiple testing (four comparisons). Differences were considered statistically significant when $P<0 \cdot 05$.

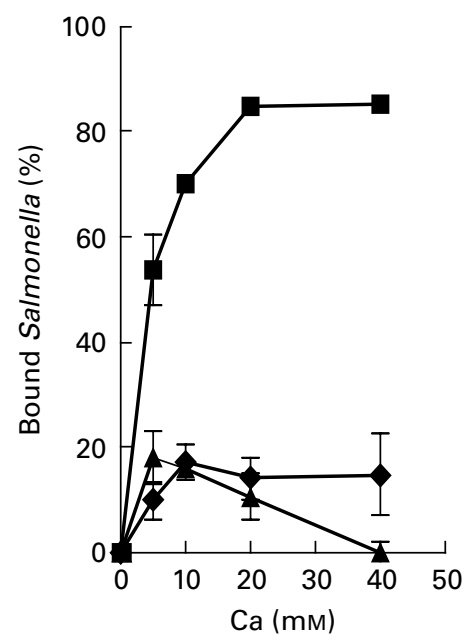

Fig. 2. Percentage of Salmonella bound to amorphous or crystalline Calcium phosphate or ionic $\mathrm{Ca}$. Results are expressed as means and standard deviations. Values are means of triplicate incubations. Standard deviations are either shown or smaller than symbols. $\mathbf{\square}$, Amorphous calcium phosphate; ionic $\mathrm{Ca} ; \mathbf{\Lambda}$, crystalline calcium phosphate. 


\section{Results}

In vitro experiments

Solubility of calcium salts and binding of Salmonella. Solubility of the different $\mathrm{Ca}$ salts was determined at $\mathrm{pH}$ $2-7$ (Fig. 1), representing the $\mathrm{pH}$ range in the gastrointestinal tract. All the $\mathrm{Ca}$ salts were soluble at lower (gastric) $\mathrm{pH}$. Calcium chloride was completely soluble at all $\mathrm{pH}$ levels tested. The other $\mathrm{Ca}$ salts were most soluble at $\mathrm{pH} 5-7$.

Freshly formed amorphous calcium phosphate was capable of binding Salmonella. In contrast, crystalline calcium phosphate and ionic Ca showed almost no binding capacity (Fig. 2).

\section{In vivo experiment}

Calcium excretion and composition of the intestinal microbiota. Before infection, faecal $\mathrm{Ca}$ excretion (as a percentage of total intake) was higher in all Casupplemented groups compared to the low-Ca control group. Urinary $\mathrm{Ca}$ excretion was lower in the calcium phosphate and milk Ca group compared to the low-Ca control group (Fig. 3).

Before infection with Salmonella, no differences in faecal lactobacilli were observed between the diet groups. Calcium phosphate and milk $\mathrm{Ca}$ reduced the number of enterobacteria compared to the low-Ca control group (Fig. 4).

Food intake and body weight. Food consumption before infection was approximately $22 \mathrm{~g}$ and did not differ between the diet groups. During the first week after infection, mean food intake was significantly higher

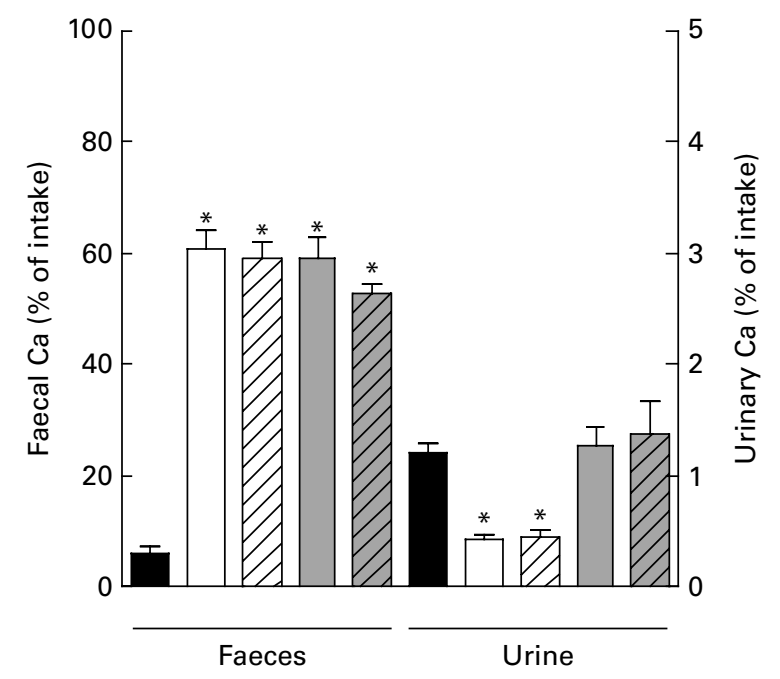

Fig. 3. Faecal and urinary $\mathrm{Ca}$ excretion (\% of intake) before infection of the rats. Ca was determined by inductively coupled plasma-atomic emission spectrophotometry. Results are expressed as means with their standard errors ( $n 7$ in the calcium phosphate group and $n 8$ in the other groups). 口, Low-Ca control; $\square$, calcium phosphate; $\mathbb{Z}$, milk $\mathrm{Ca}$; $\square$, calcium carbonate; $\mathbb{Z}$, calcium chloride. ${ }^{*}$ The indicated group is significantly different from the low-Ca control group $(P<0.05)$. in the calcium phosphate group compared to the low-Ca group (Fig. 5(a)).

Body weight gain before infection was similar in all the diet groups. However, after infection average body weight gain was significantly higher in the calcium phosphatesupplemented group (Fig. 5(b)).

Intestinal permeability. Due to ad libitum feeding of the animals, daily dietary intake of Cr-EDTA (marker for intestinal permeability) differed between days and animals. Therefore, urinary Cr-EDTA excretion was calculated as a percentage of total daily dietary intakes. Urinary Cr-EDTA in the low-Ca control group strongly increased after infection, reaching a maximum at day 5 (Fig. 6). Infection-induced intestinal permeability was lower in all Ca-supplemented groups compared to the low-Ca control group. However, the kinetics of urinary Cr-EDTA was similar in all the diet groups and illustrate Salmonellainduced damage to the intestinal barrier.

Persistence of Salmonella. All the Ca-supplemented groups decreased persistence of Salmonella, as indicated by decreased faecal excretion of Salmonella with time (Fig. 7). In contrast, rats fed the low-Ca diet continued to excrete high Salmonella numbers in their faeces.

\section{Discussion}

The present study shows that besides calcium phosphate, also milk $\mathrm{Ca}$, calcium carbonate and calcium chloride are able to enhance the resistance to infection, as indicated by the reduction of infection-induced intestinal permeability and the decreased persistence of Salmonella in

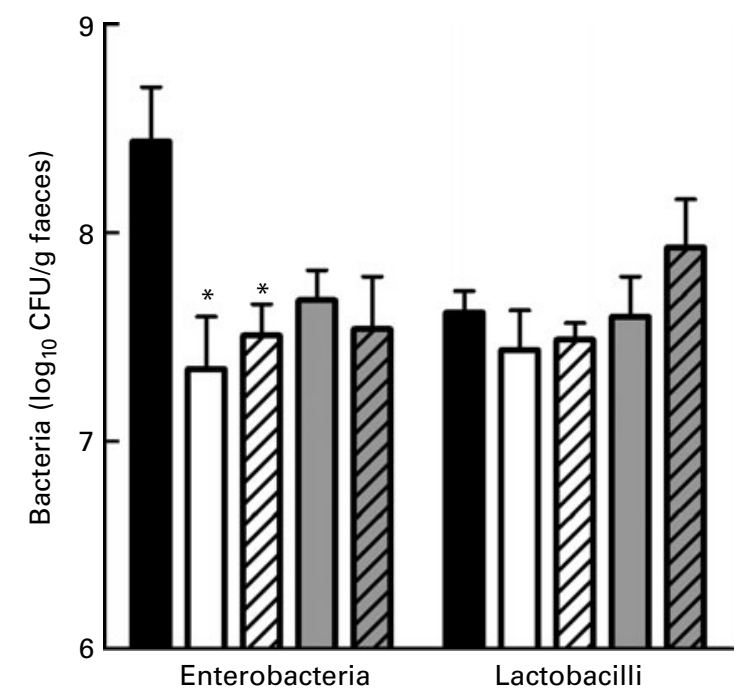

Fig. 4. Effect of dietary Ca supplementation on enterobacteria and lactobacilli in faecal samples collected before Salmonella infection of the rats. Lactobacilli were cultured anaerobically on Rogosa agar and enterobacteria were cultured aerobically on Levine EMB agar. Results are expressed as means with their standard errors ( $n 7$ in the calcium phosphate group and $n 8$ in the other groups). $\mathbf{\square}$, Low-Ca control; $\square$, calcium phosphate; milk $\mathrm{Ca}$; $\square$, cal-

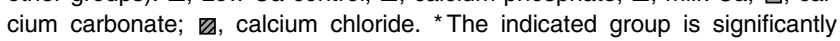
different from the low-Ca control group $(P<0.05)$. CFU, colony-forming units. 

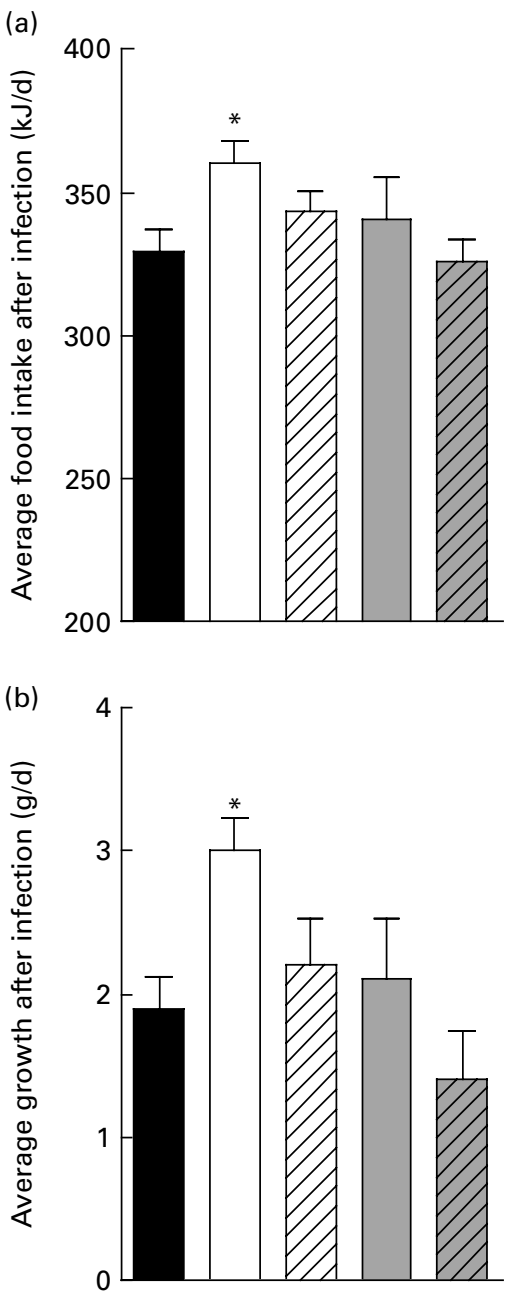

Fig. 5. Effect of dietary Ca supplementation on mean food intake (a) and growth of the rats (b) after oral administration of $1 \times 10^{9}$ colony-forming units of Salmonella enteritidis. Results are expressed as means ( $n 7$ in the calcium phosphate group and $n 8$ in the other groups). $\square$, Low-Ca control; $\square$, calcium

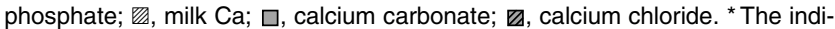
cated group is significantly different from the low-Ca control group $(P<0.05)$.

time. The calcium phosphate-induced improvement of intestinal resistance to Salmonella is in agreement with earlier studies ${ }^{(12,23,24)}$.

Protection against Salmonella by direct binding of Salmonella to the amorphous calcium phosphate complex seems a likely explanation for the observed protective effect. The amorphous calcium phosphate complex is formed in the human ${ }^{(25,26)}$ and rat ${ }^{(27)}$ proximal small intestine from soluble ionic $\mathrm{Ca}$ together with phosphate originating from the diet. The amorphous calcium phosphate complex could be formed in vivo with all tested Ca salts; soluble ionic $\mathrm{Ca}$ was present after gastric passage in vitro, Ca was abundantly present in the intestinal lumen (as indicated by the faecal Ca excretion), and all the diets contained phosphate (originating from acid casein). The in vitro experiments also showed that amorphous calcium phosphate, in contrast to crystalline calcium phosphate and ionic $\mathrm{Ca}$, was able to bind Salmonella.

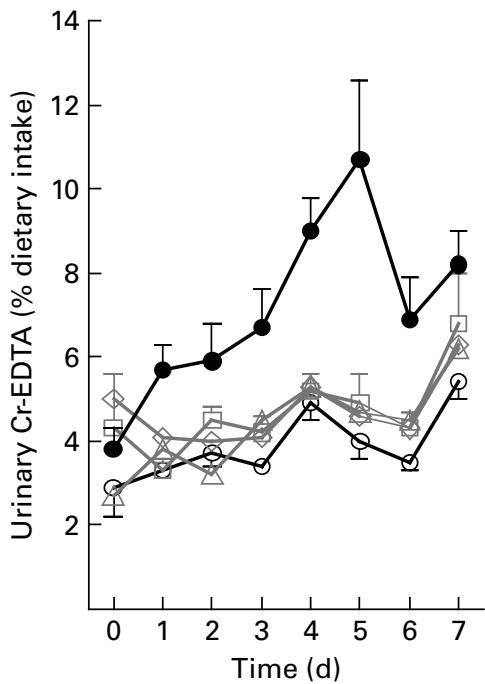

Fig. 6. Effects of dietary Ca supplementation on urinary Cr-EDTA excretion in rats. Daily urinary $\mathrm{Cr}$-EDTA excretion is expressed as $\%$ of dietary intake. Rats were challenged with $1 \times 10^{9}$ colony-forming units of Salmonella enteritidis on day 0 . Cr-EDTA was analysed by inductively coupled plasmaatomic emission spectrophotometry. Results are expressed as means with their standard errors ( $n 7$ in the calcium phosphate group and $n 8$ in the other groups). ๑, Low-Ca control; $\bigcirc$, calcium phosphate; $\square$, milk $\mathrm{Ca}$; $\diamond$, calcium carbonate; $\Delta$, calcium chloride. All the Ca-supplemented groups were significantly different from the low-Ca control group $(P<0.05)$.

Binding of Salmonella to the amorphous calcium phosphate complex in the intestinal lumen may subsequently prevent mucosal attachment of these pathogens and inhibit infection-induced pathology. This interaction may also

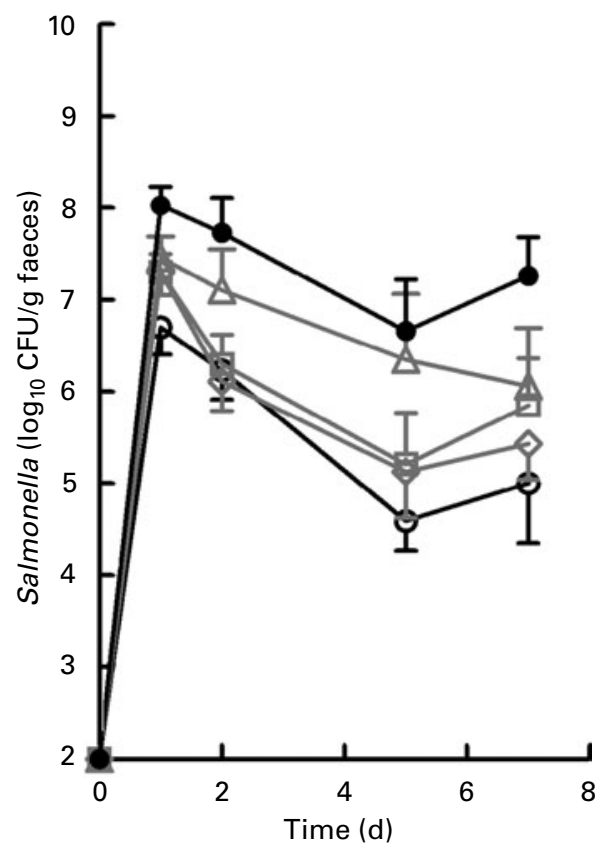

Fig. 7. Effect of dietary Ca supplementation on faecal Salmonella excretion with time. Rats orally received $1 \times 10^{9}$ colony-forming units (CFU) of Salmonella enteritidis on day 0 . Results are expressed as means with their standard errors ( $n 7$ in the calcium phosphate group and $n 8$ in the other groups). •, Low-Ca control; $\bigcirc$, calcium phosphate; $\square$, milk $\mathrm{Ca} ; \diamond$, calcium carbonate; $\triangle$, calcium chloride. All the Ca-supplemented groups were significantly different from the low-Ca control group $(P<0.05)$. 
play a role in the observed dietary Ca-induced reduction in faecal enterobacteria in the present study.

Besides direct binding of Salmonella during gut transit, amorphous calcium phosphate can also precipitate cytotoxic luminal components, such as bile acids and fatty acids, within the intestinal lumen ${ }^{(25-27)}$. Binding of these surfactants can subsequently prevent epithelial cell damage and compensatory epithelial hyperproliferation $^{(26,28)}$, and may protect the intestinal barrier ${ }^{(25,26,29)}$. In fact, in the present study, all the tested dietary Ca salts prevented the infection-related increase in intestinal permeability.

The protective effects of dietary $\mathrm{Ca}$ are not restricted to Salmonella. We have previously shown that dairy Ca strongly improves the resistance to enterotoxigenic Escherichia coli in both rats and human subjects. To follow-up, a large intervention trial is currently being performed to determine whether milk $\mathrm{Ca}$ can protect against acute infectious diarrhoea in Indonesian children.

Are the dietary concentrations of $\mathrm{Ca}$ in our animal studies relevant for the human diet? In general, dietary $\mathrm{Ca}$ intake in the Western world ranges from 600 to $1100 \mathrm{mg} / \mathrm{d}^{(30,31)}$. Assuming a daily food intake of $500 \mathrm{~g}$ dry weight per $d$, the average concentration in the food would be $1 \cdot 2-2 \cdot 2 \mathrm{~g} / \mathrm{kg}$. The animal diets in this mechanistic study contained Ca levels of $0.8 \mathrm{~g} / \mathrm{kg}(20 \mathrm{mmol} / \mathrm{kg}$ diet $)$ and $4.0 \mathrm{~g} / \mathrm{kg}$ (diets supplemented to $100 \mathrm{mmol} / \mathrm{kg}$ ), and therefore represent conditions slightly lower and higher than normal human intake.

Overall, the present study showed that several dietary Ca salts (calcium phosphate, milk Ca, calcium carbonate, calcium chloride) are protective in improving resistance to Salmonella infection. This protective effect might be explained by binding of Salmonella (thereby preventing mucosal attachment of Salmonella) and/or binding of cytotoxic luminal components (thereby protecting the intestinal barrier). In view of the growing resistance of pathogenic bacteria to antibiotic drugs, modulation of host resistance to intestinal infections by dietary Ca might be an attractive approach.

\section{Acknowledgements}

The authors thank the workers at the laboratory animal facility of Wageningen University for technical assistance. M. H. C. S. and E. E. are employees of FrieslandCampina Domo (Zwolle, The Netherlands) that is a producer of milk $\mathrm{Ca}$ used in the present study. The remaining authors declare no conflict of interest. No external funding, apart from the authors' institutions, was available for the present study. All the authors contributed to the preparation of the paper and agreed with the submitted manuscript content. S. J. M. t. B., J. S., M. H. C. S. and E. E. designed the research; E. V. d. M. and A. S. performed the research and analysed the data; and. S. J. M. t. B., J. S. and I. M. J. B.-O. drafted the paper.

\section{References}

1. Schlundt J (2001) Emerging food-borne pathogens. Biomed Environ Sci 14, 44-52.

2. Todd EC (1997) Epidemiology of foodborne diseases: a worldwide review. World Health Stat Q 50, 30-50.

3. Mead PS, Slutsker L, Dietz V, et al. (1999) Food-related illness and death in the United States. Emerg Infect Dis 5, 607-625.

4. World Health Organization (2005) Drug-Resistant Salmonella. Factsheet no. 139. Geneva: WHO.

5. Osterholm MT (2000) Emerging infections-another warning. $N$ Engl J Med 342, 1280-1281.

6. Bovee-Oudenhoven IM, ten Bruggencate SJ, Lettink-Wissink ML, et al. (2003) Dietary fructo-oligosaccharides and lactulose inhibit intestinal colonisation but stimulate translocation of Salmonella in rats. Gut 52, 1572-1578.

7. Sprong RC, Hulstein MF \& Van der Meer R (1999) High intake of milk fat inhibits intestinal colonization of Listeria but not of Salmonella in rats. J Nutr 129, 1382-1389.

8. Bovee-Oudenhoven IM, Wissink ML, Wouters JT, et al. (1999) Dietary calcium phosphate stimulates intestinal lactobacilli and decreases the severity of a Salmonella infection in rats. J Nutr 129, 607-612.

9. Kleessen B, Sykura B, Zunft HJ, et al. (1997) Effects of inulin and lactose on fecal microflora, microbial activity, and bowel habit in elderly constipated persons. Am J Clin Nutr $\mathbf{6 5}$, $1397-1402$

10. Shoda R, Mahalanabis D, Wahed MA, et al. (1995) Bacterial translocation in the rat model of lectin induced diarrhoea. Gut 36, 379-381.

11. Bovee-Oudenhoven IM, Termont DS, Weerkamp AH, et al. (1997) Dietary calcium inhibits the intestinal colonization and translocation of Salmonella in rats. Gastroenterology 113, 550-557.

12. Bovee-Oudenhoven IM, Lettink-Wissink ML, Van Doesburg $\mathrm{W}$, et al. (2003) Diarrhea caused by enterotoxigenic Escherichia coli infection of humans is inhibited by dietary calcium. Gastroenterology 125, 469-476.

13. Govers MJ, Termont DS \& Van der Meer R (1994) Mechanism of the antiproliferative effect of milk mineral and other calcium supplements on colonic epithelium. Cancer Res 54, 95-100.

14. Govers MJ, Termont DS, Van Aken GA, et al. (1994) Characterization of the adsorption of conjugated and unconjugated bile acids to insoluble, amorphous calcium phosphate. J Lipid Res 35, 741-748.

15. Calvo MS (1993) Dietary phosphorus, calcium metabolism and bone. J Nutr 123, 1627-1633.

16. Reeves PG, Nielsen FH \& Fahey GC Jr (1993) AIN-93 purified diets for laboratory rodents: final report of the American Institute of Nutrition ad hoc writing committee on the reformulation of the AIN-76A rodent diet. J Nutr 123, 1939-1951.

17. Ten Bruggencate SJM, Bovee-Oudenhoven IMJ, LettinkWissink MLG, et al. (2003) Dietary fructo-oligosaccharides dose-dependently increase translocation of Salmonella in rats. J Nutr 133, 2313-2318.

18. Giaffer MH, Holdsworth CD \& Duerden BI (1991) The assessment of faecal flora in patients with inflammatory bowel disease by a simplified bacteriological technique. J Med Microbiol 35, 238-243.

19. Bjarnason I, Maxton D, Reynolds AP, et al. (1994) Comparison of four markers of intestinal permeability in control subjects and patients with coeliac disease. Scand J Gastroenterol 29, 630-639.

20. Oman H, Blomquist L, Henriksson AE, et al. (1995) Comparison of polysucrose 15000 , 51Cr-labelled ethylenediaminetetraacetic acid, and 14C-mannitol as markers of intestinal permeability in man. Scand J Gastroenterol 30, 1172-1177. 
21. Binnerts W, Van het Klooster A \& Frens AM (1968) Soluble chromium indicator measured by atomic absorption in digestion experiments. Vet $\operatorname{Rec} \mathbf{8 2}, 470$.

22. Bovee-Oudenhoven IM, Termont DS, Heidt PJ, et al. (1997) Increasing the intestinal resistance of rats to the invasive pathogen Salmonella enteritidis: additive effects of dietary lactulose and calcium. Gut 40, 497-504.

23. Ten Bruggencate SJ, Bovee-Oudenhoven IM, Lettink-Wissink ML, et al. (2004) Dietary fructo-oligosaccharides and inulin decrease resistance of rats to Salmonella: protective role of calcium. Gut 53, 530-535.

24. van Ampting MT, Rodenburg W, Vink C, et al. (2009) Ileal mucosal and fecal pancreatitis associated protein levels reflect severity of Salmonella infection in rats. Dig Dis Sci 22 (Epublication ahead of print version 22 January 2009).

25. Govers MJ, Termont DS, Lapre JA, et al. (1996) Calcium in milk products precipitates intestinal fatty acids and secondary bile acids and thus inhibits colonic cytotoxicity in humans. Cancer Res 56, 3270-3275.

26. Van der Meer R, Welberg JW, Kuipers F, et al. (1990) Effects of supplemental dietary calcium on the intestinal association of calcium, phosphate, and bile acids. Gastroenterology 99 , 1653-1659.

27. Bovee-Oudenhoven IM, Wissink ML, Wouters JT, et al. (1999) Dietary calcium phosphate stimulates intestinal lactobacilli and decreases the severity of a Salmonella infection in rats. J Nutr 129, 607-612.

28. Sesink AL, Termont DS, Kleibeuker JH, et al. (2001) Red meat and colon cancer: dietary haem-induced colonic cytotoxicity and epithelial hyperproliferation are inhibited by calcium. Carcinogenesis 22, 1653-1659.

29. Govers MJ \& Van der Meer R (1993) Effects of dietary calcium and phosphate on the intestinal interactions between calcium, phosphate, fatty acids, and bile acids. Gut 34, 365-370.

30. Buchowski MS, Semenya J \& Johnson AO (2002) Dietary calcium intake in lactose maldigesting intolerant and tolerant African-American women. J Am Coll Nutr 21, 47-54.

31. Lovejoy JC, Champagne CM, Smith SR, et al. (2001) Ethnic differences in dietary intakes, physical activity, and energy expenditure in middle-aged, premenopausal women: the Healthy Transitions Study. Am J Clin Nutr 74, 90-95. 